\title{
Desempenho de estudantes de Ensino Médio em um teste sobre conhecimentos básicos de cinemática e transformação de unidades
}

A

\section{Performance of High School students in a test on basic kinematics and unit transformation}

\author{
Revista
}

Educar Mais

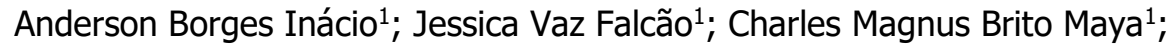
Guilherme Frederico Marranghello ${ }^{1}$; Pedro Dorneles ${ }^{1}$

\begin{abstract}
RESUMO
A partir do alto índice de reprovações e abando nos componentes curriculares, de primeiro semestre, de cálculo e Física do campus Bagé da Universidade Federal do Pampa no Programa Institucional de Bolsa de Iniciação à Docência (PIBID) da licenciatura em Física foi elaborado e aplicado um teste sobre conhecimentos básicos de transformação de unidades, Cinemática e interpretação de gráficos. O teste foi aplicado a 944 estudantes dos três anos de ensino médio de todas as escolas estaduais do perímetro urbano de Bagé-RS. Os resultados evidenciam uma realidade que merece muita atenção, pois o percentual de acertos foi menor que $30 \%$. Apesar de se identificar uma diferença estatisticamente significativa entre os dados do primeiro e terceiro ano e entre os do primeiro e segundo ano tais diferenças não são expressivas, pois os percentuais de acertos do primeiro, segundo e terceiro ano foram: $27 \%, 29 \%$ e $30 \%$ respectivamente.
\end{abstract}

Palavras-chave: Ensino Médio; Teste de conhecimento; Ensino de Física; Gráficos.

\begin{abstract}
Based on the high failure rate and on the first semester, calculus and physics components of the Universidade Federal do Pampa - Campus Bagé in the Institutional Program for Teaching Initiation Scholarship (PIBID) of the Physics Degree, a test was designed and applied. on basic knowledge of physics and unit transformation. The test was applied to 944 students from the three years of high school of all state schools in the urban perimeter of Bagé-RS. The results show a reality that deserves much attention, because the percentage of correct answers was less than 30\%. Although a statistically significant difference was identified between the first and third year data and between the first and second year data, such differences are not significant, since the percentages of first, second and third year hits were: 27\%, 29\%. and 30\% respectively.
\end{abstract}

Keywords: High school, Knowledge test, Physics teaching, Graphics.

\footnotetext{
${ }^{1}$ UNIPAMPA - Universidade Federal do Pampa, Bagé/RS - Brasil.
} 


\section{INTRODUÇÃO}

A importância de estudantes possuírem conhecimentos prévios sobre transformação de unidades e conceitos básicos de cinemática, incluindo análise gráfica, se justifica pelo fato de serem conteúdos iniciais do componente curricular de Física universitária de cursos de ciências exatas. Segundo Araujo, Veit e Moreira (2004) uma das habilidades requeridas para a compreensão de conteúdos de Física é a interpretação de gráficos, porém muitas vezes pouca compreendida por estudantes. Tal dificuldade pode ser um indicativo para os baixos índices de aprovações no componente de Física de primeiro semestre de cursos do campus Bagé da Universidade Federal do Pampa. Em um levantamento realizado no primeiro semestre de 2017 pelos autores do presente trabalho, a partir de dados institucionais, foi encontrado que apenas 48 estudantes foram aprovados de um total de 314 alunos matriculados no componente de Física de primeiro semestre dos cursos de Engenharia de Alimentos, Engenharia de Energia, Engenharia de Produção, Engenharia Química e Licenciatura em Física do Campus Bagé da Universidade Federal

Dada tal situação, um grupo de bolsistas do Programa Institucional de Bolsas de Iniciação à Docência (PIBID) do subprojeto Física da Unipampa campus Bagé desenvolveu ações em escolas públicas da região da cidade de Bagé-RS, com o objetivo de identificar os conhecimentos básicos dos alunos do ensino médio (EM). Para isso, iniciou-se uma revisão da literatura para a identificação de trabalhos sobre testes de conhecimento em um período de 11 anos (2007-2017), com o propósito de propiciar o embasamento teórico para elaboração e aplicação de um teste sobre conhecimentos básicos de Física e Matemática.

Após as etapas de validação de conteúdo e de fidedignidade o teste foi aplicado em escolas estaduais do município de Bagé-RS, atingindo um total de 944 estudantes de ensino médio.

No presente trabalho buscamos analisar se ao longo dos três anos do Ensino Médio os estudantes apresentam um desempenho crescente e se ao final do Ensino Médio (terceiro ano) são capazes de resolverem problemas simples de transformação de unidades (por exemplo: transformações em unidades de comprimento, área, volume, velocidade e tempo), de movimentos retilíneos e de interpretação de gráficos de posição e velocidade.

\section{REVISÃO DA LITERATURA}

Definida a problemática a ser tratada no presente trabalho, inicialmente realizamos uma revisão da literatura focada em testes de conhecimentos e possibilidades didáticas sobre dificuldades de aprendizagem de alunos do ensino médio sobre conceitos introdutórios de Física. A partir desse enfoque foi feito um levantamento de artigos publicados com tais objetivos, sendo realizadas pesquisas em 10 revistas de Ensino de Física², sendo elas:

1. Revista Brasileira de Ensino de Física,

2. Revista Brasileira de Ensino de Ciência e Tecnologia,

\footnotetext{
${ }^{2}$ Dada a impossibilidade de pesquisa individual nas centenas de revistas que publicam trabalhos sobre Ensino, os autores escolheram essas 10 revistas, mas salientam que com o crescente número de revistas não se trata de uma seleção que inclui exclusivamente as principais revista da área.
} 
3. Investigações em Ensino de Ciências,

4. Ciência \& Educação,

5. A Física na Escola,

6. Caderno Brasileiro de Ensino de Física,

7. Revista Brasileira de Física Tecnológica Aplicada,

8. Revista Electrónica de Enseñanza de las Ciencias,

9. Experiências em Ensino de Ciências,

10. ACTIO: Docência em Ciências.

Para a seleção dos artigos foi realizada uma leitura dos resumos e palavras-chaves de todos os volumes das revistas citadas, no período de 2007 até 2017.

Em relação a trabalhos com a divulgação de testes, não encontramos nenhum trabalho no período de 2007 a 2017. Contudo, encontramos diversos artigos abordando propostas que procuram a superação de dificuldades na aprendizagem dos alunos de ensino médio, a saber: Costa, Almeida, Lopes (2015); Gomes, Garcia (2014); Bulegon, Tarouco (2015); Dias, Novikoff, Souza (2011); Machado, Pinheiro (2010); Lima, Tenório, Bastos (2010); Coutinho, Folmer, Puntel (2014); Machado, Marques, Silva (2016); Laburú, Barros (2009); Gomes, Borges, Justi (2008); Oliveira, Moreira (2016); Santarosa, Moreira (2011); Belançon (2017); Oliveira, Araujo, Veit (2016); Parisoto, Moreira, Kilian (2016); Ricardo, Freire (2007); Lunkes, Rocha Filho (2011); Costa Junior, Rodrigues, Silva, Gomes, Assis (2017); Gerab, Valério (2014); Martins, Abelha, Costa, Roldão (2011); Proença, Pirola (2011); Santos, Curi (2012); Baccon, Arruda (2010).

Como não identificamos nenhum artigo sobre testes de conhecimento, refizemos nossa pesquisa na Revista Brasileira de Ensino de Física. A escolha por essa revista se deu pelo fato de ser Qualis A1 e ser uma revista de referência para a comunidade de pesquisadores em Ensino de Física. Ao acessar o sistema de busca da revista utilizamos a palavra "teste" como descritor. Assim, encontramos 25 trabalhos que, após uma análise de todo o conteúdo dos artigos, selecionamos dois trabalhos, sendo eles: Bernal, Yamamoto (2002) e Araujo, Veit, Moreira (2004).

Em relação aos testes apresentados nos trabalhos selecionados, destacamos o grau de dificuldade deles, pois foram aplicados com alunos dos primeiros anos de universidades federais, sendo realidades distintas da população-alvo do presente artigo. Diante disso, optamos por criar um teste com questões mais simples, a partir das questões de tais testes. A seguir, descreveremos brevemente os testes encontrados.

Bernal e Yamamoto (2002) buscaram identificar as principais dificuldades conceituais de Física de alunos ingressantes de Engenharia, utilizando um teste o qual foi desenvolvido nos EUA em uma escola do Arizona e na Universidade de Harvard, o mechanics baseline test (MBT), sendo um teste considerado como simples pelos autores, no qual argumentam a não necessidade de o aluno possuir conhecimentos mais aprofundados de Matemática para a solução dos problemas, mas sendo fundamental que os alunos dominem conceitos básicos, tais como álgebra simples, ao qual não se necessita de uma matemática bem elaborada, com isso dispensando o uso de calculadora. 
Em uma primeira aplicação, o teste foi aplicado em uma universidade não identificada pelos autores no artigo no segundo semestre de 1999, com 771 alunos de Física I e, posteriormente, no segundo semestre de 2000, com 814 alunos também de Física I, ambos aplicados nas primeiras semanas de aula. Após a aplicação, os resultados foram comparados com os dados das aplicações do teste em Harvard e na escola do Arizona, realizando uma comparação dos dados brasileiros com os norteamericanos. As questões foram separadas em áreas determinadas por conteúdos abordados no ensino médio, como a cinemática (movimento linear, aceleração constante, aceleração média, deslocamento por integração), movimento curvilíneo (aceleração tangencial, aceleração normalmente, uso da expressão $a_{n}=V^{2} / r$ ), dinâmica (primeira lei, segunda lei, dependência com a massa, terceira lei, princípio da superposição, trabalho-energia, conservação da energia, impulsomomento linear e conservação do momento linear) e forças específicas (queda livre gravitacional e atrito). Os resultados não apresentaram uma diferença estatisticamente significativa entre as duas turmas, de 1999 e de 2000, com significância igual ou menor de 0,05. Já os dados da comparação entre escola do Arizona com a turma de 1999 mostraram haver uma diferença significativa entre os grupos de alunos e, se comparados com Harvard, a diferença se mostra ainda maior, denotando haver uma diferença estatisticamente significativa com os alunos brasileiros e evidenciando que os ingressantes de Harvard estão mais bem preparados que os brasileiros.

O artigo de Araujo, Veit, Moreira (2004) está embasado em um trabalho que busca propiciar condições favoráveis à aprendizagem significativa sobre gráficos da cinemática. Esse artigo foi construído tendo como objeto de pesquisa com calouros da UFRGS no segundo semestre letivo de 2002. O teste utilizado foi adaptado do teste TUG-K (teste sobre o entendimento de gráficos da cinemática), o qual tem por objetivo identificar a compreensão na interpretação de gráficos da cinemática. Os autores justificam que gráficos são utilizados para interpretações físicas que facilitam uma obtenção mais detalhada da situação estudada.

Os autores usaram um software de modelagem para propiciar uma maior interação dos estudantes com o processo de construção e análise do conhecimento científico, permitindo que compreendam melhor os modelos físicos e discutam o contexto. O software de escolha de Araujo, Veit, Moreira (2004) foi o Modellus ${ }^{3}$. Uma das habilidades requeridas para a compreensão de conteúdos de Física é a construção e elaboração de gráficos, em que grande parte da informação pode ser resumida e muito se pode extrair, entretanto, tal ideia é pouco compreendida pelos alunos. Todos os participantes do estudo já tinham sido expostos a conteúdos de cinemática anteriormente, sendo assim, foi realizada a aplicação do teste.

Avaliamos algumas questões do teste aplicado por Araujo, Veit, Moreira (2004) para selecionar algumas e, após as avaliarmos, decidimos que seriam usados como modelos para a elaboração de novas questões para ser incrementadas em nosso teste.

\section{TESTE}

Uma primeira versão do teste (teste-piloto) foi elaborada com 16 questões de múltipla escolha (contendo quatro alternativas) e aplicada em uma escola estadual da cidade de Bagé-RS, servindo

${ }^{3}$ Disponível em https://docente.ifrn.edu.br/alessandrorolim/informatica-aplicada-a-fisica/software-modellus4.01/view. Acessado em 17 de setembro de 2019. 
de parâmetro para futuras reformulações a partir da validação de conteúdo e de fidedignidade. Essa primeira versão do teste foi aplicada para um grupo de 17 alunos.

A validação de conteúdo do teste foi realizada com quatro professores, sendo três deles atuais docentes do ensino superior e um de escola pública. A ficha de avaliação continha uma introdução inicial da proposta do teste. As análises dos docentes sobre a validação de conteúdo do teste apresentaram sugestões de alterações, dentre elas uma mudança no título do teste; dentre os quatro professores, três destacaram especificamente mudanças nas questões em relação ao conhecimento básico de Física, havendo muitas questões de transformação de unidade. Na visão dos avaliadores estávamos focando mais Matemática do que Física com o número significativo de questões envolvendo transformação de unidades. Reduzimos o número de questões sobre essa abordagem, mas optamos por manter algumas questões, por notar em experiências anteriores que esse é um dos fatores em relação ao qual os estudantes apresentam mais dificuldades. Outras observações foram em relação às expressões redigidas de modo ambíguo ao longo do teste e alternativas com textos inadequados. Após analisar as opiniões dadas dos avaliadores, como dito anteriormente, decidiu-se manter algumas questões por uma necessidade já especificada, mas também ocorreram mudanças as quais os avaliadores apontaram.

O coeficiente de fidedignidade do teste foi calculado a partir do coeficiente alfa de Cronbach (CRONBACH, 1951 apud SILVEIRA, MOREIRA e AXT, 1989), com uma amostra de 300 respostas de estudantes de primeiro, segundo e terceiro ano do EM (100 respostas de cada ano). O resultado encontrado de 0,81 foi satisfatório, pois, segundo Silveira, Moreira e Axt (1989), coeficientes acima de 0,8 são valores aceitáveis em testes da área de Educação.

A versão final do teste ficou composta por 15 itens (Apêndice A), dividida entre:

- $\quad$ nove questões (1-9) sobre Sistema Internacional de Medidas (SI) e notação científica;

- $\quad$ três questões (10-12) envolvendo noções básicas de cinemática;

- $\quad$ três questões (13-15) sobre interpretação de gráficos da cinemática.

Os conteúdos de SI, notação científica, cinemática e interpretação de gráficos da cinemática foram escolhidos por serem conteúdos que constituem tradicionalmente as primeiras semanas de aula dos estudantes do componente de Física de primeiro semestre de um curso universitário de Ciências Exatas e ser algo que pode ser considerado como um conhecimento prévio necessário para vários conteúdos que são abordados ao longo da formação universitária.

\section{APLICAÇÃO DO TESTE}

Anteriormente à aplicação dos testes foi apresentada para as direções das escolas estaduais da cidade de Bagé-RS uma carta de apresentação relatando o propósito e a motivação da aplicação do teste em suas escolas e como os dados seriam utilizados após a aplicação nas turmas de ensino médio. Após todas as autorizações serem aprovadas pelos(as) diretores(as) foi então criada uma tabela de aplicações junto às escolas para se prever um horário aceitável tanto aos aplicadores como aos professores e seus alunos. 
Subsequentemente à aprovação e horários marcados, iniciou-se a aplicação dos testes nas escolas, em turmas do primeiro ao terceiro ano do ensino médio, dentre as nove escolas nas quais foram aplicados os testes em 944 alunos, sendo 427 alunos do primeiro ano, 240 do segundo ano e 277 do terceiro ano, com média de tempo de 30 minutos para cada aplicação do teste. Ao longo das aplicações do teste nas escolas, devido à aproximação do fim do ano letivo das escolas, viu-se necessário o apoio de alguns bolsistas do PIBID-Física, por meio de uma solicitação via e-mail, sendo que alguns foram voluntários para colaborar com as aplicações nas escolas, sendo designados para escolas em diferentes turnos para assim poder concluir a aplicação do teste antes do recesso dos alunos.

Durante as aplicações, encontraram-se algumas dificuldades que foram, ao longo do trabalho proposto, vistas como variáveis que, de certa forma, foram inconvenientes tanto por parte dos aplicadores do teste como em relação às escolas, que cederam um pouco de seu tempo para colaborar com a pesquisa. O período de aplicação ocorreu próximo ao recesso das escolas e alguns atrasos nas provas de certas turmas colaboraram com o atraso da aplicação do teste, mas, com paciência, persistência e colaboração de ambas as partes puderam-se satisfazer os objetivos, tanto na aplicação do teste como na liberação dos alunos para suas férias. Mesmo assim, em alguns casos não foi possível conseguir uma aplicação completa em algumas escolas.

\section{RESULTADOS E DISCUSSÃO}

$\mathrm{Na}$ tabela 1 encontram-se os dados gerais da pesquisa realizada. Nessa tabela encontra-se a média geral e o desvio-padrão da medida e da média envolvendo todas as turmas de primeiro a terceiro ano e elas divididas por anos, I representando o primeiro ano; II o segundo; e III o terceiro.

Tabela 1: dados gerais.

\begin{tabular}{|c|c|c|c|c|}
\hline Grupo & $\begin{array}{c}\text { N (número de } \\
\text { respondentes) }\end{array}$ & $\begin{array}{c}\text { Média de acertos } \\
\text { das 15 questões }\end{array}$ & $\begin{array}{c}\text { Desvio- } \\
\text { padrão da } \\
\text { medida }\end{array}$ & $\begin{array}{c}\text { Desvio- } \\
\text { padrão da } \\
\text { média }\end{array}$ \\
\hline Geral & 944 & 4,31 & 2,05 & 0,07 \\
\hline I & 427 & 4,11 & 1,89 & 0,09 \\
\hline II & 240 & 4,40 & 2,13 & 0,14 \\
\hline III & 277 & 4,54 & 2,18 & 0,13 \\
\hline
\end{tabular}

Fonte: dados da pesquisa (2017).

Com o gráfico da figura 1 pode-se notar que as médias obtidas juntamente com a barra de erro (desvio padrão da média) mostram que o terceiro ano obteve um melhor aproveitamento. Em uma primeira análise se identifica um crescimento nas médias do primeiro ano ao terceiro ano. No entanto, algo pouco expressivo, pois em nenhum ano se ultrapassou a média de cinco acertos, ou seja, de 15 itens do teste não se atingiu em nenhum ano um percentual de acertos maior que $30 \%$. Cabe destacar que a evolução identificada não apresentou aumento maior que um item, pois a média do primeiro ano foi de 4,11 e do terceiro ano de 4,45 . 
Figura 1: gráfico da média geral e do primeiro, segundo e terceiro ano.

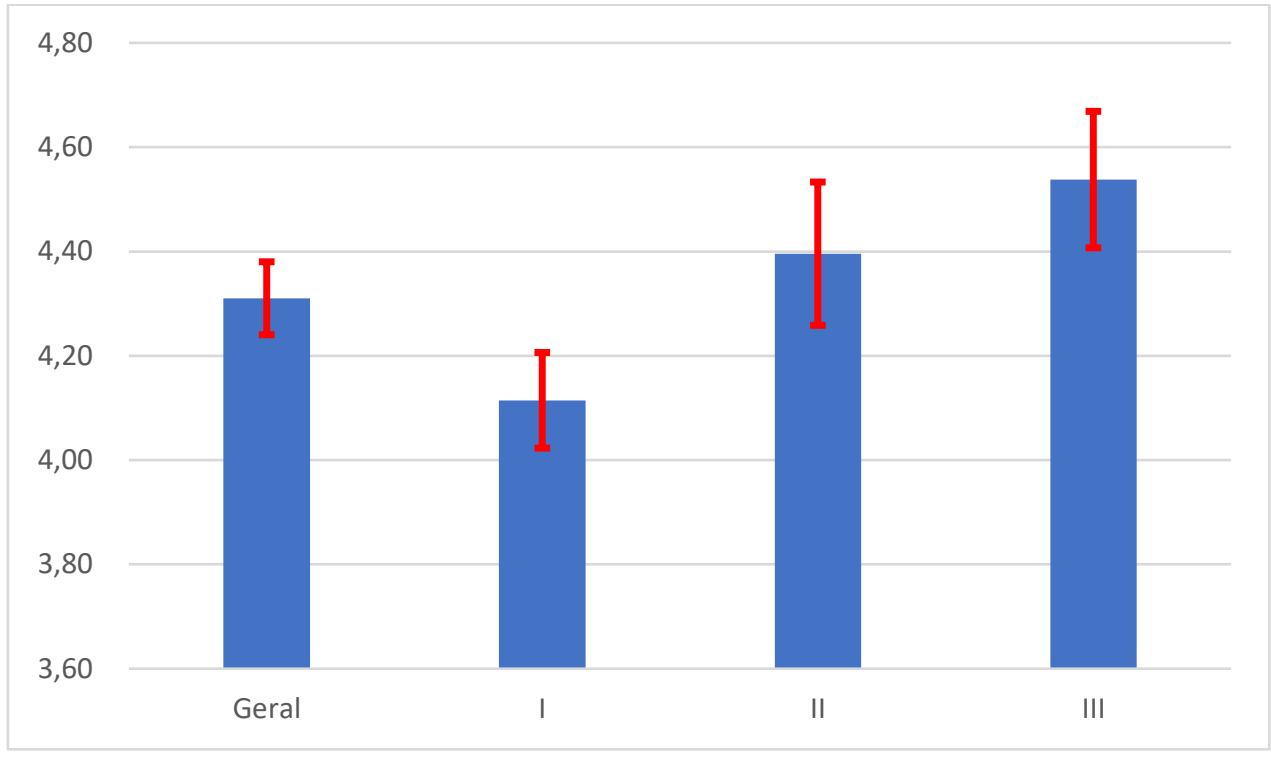

Fonte: dados da pesquisa (2017).

Apesar das diferenças entre as médias serem baixas, foi realizado um estudo sobre a significância estatística para as diferenças entre as médias de cada ano. Foi realizada uma análise estatística, com o uso do teste t de Student, conforme as comparações propostas por Silveira (2019). A interpretação da tabela 2 demonstra que foram encontradas diferenças estatísticas entre o primeiro e terceiro ano a um nível menor que 0,05 , ou seja, uma probabilidade maior que $95 \%$ de que tal diferença não tenha sido por acaso. Em relação ao segundo ano com o terceiro não foram encontradas diferenças estatisticamente significativas, enquanto entre o primeiro e o segundo ano foi encontrado um nível de significância menor que 0,1 (probabilidade maior que $90 \%$ de que tal diferença não tenha sido por acaso).

Tabela 2: comparação dos resultados entre os anos.

\begin{tabular}{|l|l|l|}
\hline Comparação & $\begin{array}{l}\text { Teste t de Student } \\
\text { (t crítico bicaudal = 1,96) }\end{array}$ & $\begin{array}{l}\text { Nível de significância } \\
\text { estatística }\end{array}$ \\
\hline I-II & 1,758 & 0,079 \\
\hline III-II & 0,747 & 0,455 \\
\hline III-I & 2,728 & 0,006 \\
\hline
\end{tabular}

Fonte: dados da pesquisa (2017).

Na tabela 3 apresenta-se o resultado do teste aplicado às turmas de primeiro a terceiro ano das escolas estaduais de Bagé-RS. Nessa tabela há os índices percentuais de cada alternativa (A, B, C e D) e destacados em negrito encontram-se os índices que correspondem às alternativas corretas, constando a porcentagem geral de questões marcadas corretamente junto com o percentual de cada questão individualmente selecionada corretamente. 
Tabela 3: dados individuais do I, I e III ano.

\begin{tabular}{|c|c|c|c|c|c|c|c|c|c|c|c|c|c|c|c|}
\hline $\begin{array}{c}\text { Questão/ } \\
\text { opção }\end{array}$ & 1 & 2 & 3 & 4 & 5 & 6 & 7 & 8 & 9 & 10 & 11 & 12 & 13 & 14 & 15 \\
\hline A I & 24 & 50 & 25 & 25 & 11 & 30 & 23 & 21 & 17 & 18 & 24 & 30 & 28 & 21 & 22 \\
\hline B I & 17 & 22 & 18 & 31 & 21 & 28 & 25 & 38 & 18 & 31 & 24 & 30 & 26 & 19 & 29 \\
\hline C I & 16 & 11 & 34 & 16 & 16 & 22 & 19 & 21 & 42 & 33 & 23 & 20 & 30 & 32 & 23 \\
\hline D I & 42 & 13 & 16 & 22 & 48 & 14 & 28 & 14 & 18 & 13 & 24 & 15 & 12 & 22 & 21 \\
\hline Em branco I & 1 & 5 & 7 & 7 & 4 & 6 & 5 & 5 & 4 & 5 & 6 & 4 & 4 & 6 & 5 \\
\hline A II & 21 & 57 & 18 & 27 & 10 & 33 & 24 & 19 & 16 & 20 & 27 & 35 & 34 & 29 & 27 \\
\hline B II & 30 & 17 & 25 & 37 & 20 & 29 & 31 & 37 & 15 & 27 & 24 & 28 & 17 & 15 & 28 \\
\hline C II & 13 & 12 & 37 & 17 & 25 & 21 & 18 & 30 & 48 & 33 & 22 & 17 & 35 & 28 & 24 \\
\hline D II & 35 & 13 & 16 & 15 & 43 & 16 & 25 & 12 & 19 & 16 & 24 & 18 & 11 & 21 & 17 \\
\hline Em branco II & 0 & 2 & 5 & 4 & 2 & 1 & 3 & 3 & 3 & 4 & 3 & 3 & 4 & 6 & 4 \\
\hline A III & 22 & 54 & 19 & 31 & 12 & 30 & 20 & 18 & 14 & 21 & 25 & 34 & 26 & 22 & 21 \\
\hline B III & 30 & 22 & 30 & 30 & 16 & 27 & 33 & 36 & 16 & 30 & 22 & 29 & 18 & 17 & 30 \\
\hline C III & 11 & 12 & 34 & 17 & 19 & 24 & 17 & 26 & 44 & 31 & 21 & 20 & 38 & 31 & 26 \\
\hline D III & 37 & 8 & 14 & 17 & 49 & 15 & 24 & 16 & 21 & 13 & 27 & 13 & 13 & 25 & 18 \\
\hline Em branco III & 0 & 4 & 4 & 5 & 4 & 4 & 7 & 4 & 4 & 5 & 5 & 5 & 5 & 6 & 5 \\
\hline PGpQ* & 24 & 53 & 23 & 19 & 47 & 28 & 29 & 25 & 44 & 30 & 22 & 29 & 12 & 23 & 24 \\
\hline PGnT** & 29 & & & & & & & & & & & & & & \\
\hline
\end{tabular}

*PGpQ: porcentagem geral por questão.

**PGnT: porcentagem geral de acertos no teste.

Fonte: dados da pesquisa (2017).

Na figura 2 consta o gráfico que apresenta a porcentagem de acertos correspondentes a cada questão do teste. Nota-se que não há nenhuma questão acima de $60 \%$ de acertos, sendo apenas três acima de $40 \%$ e duas abaixo de $20 \%$. A questão de número 2 , que contém a maior porcentagem de acertos, corresponde à transformação de unidade, que requeria do aluno a transformação de $5.000 \mathrm{~m}$ para km. A questão 13 teve a menor porcentagem de acerto; nessa questão o aluno deveria interpretar o gráfico de velocidade de um objeto em função do tempo para escolher uma alternativa sobre a relação da aplicada força versus tempo. 
Figura 2: gráfico da porcentagem de acertos por questão.

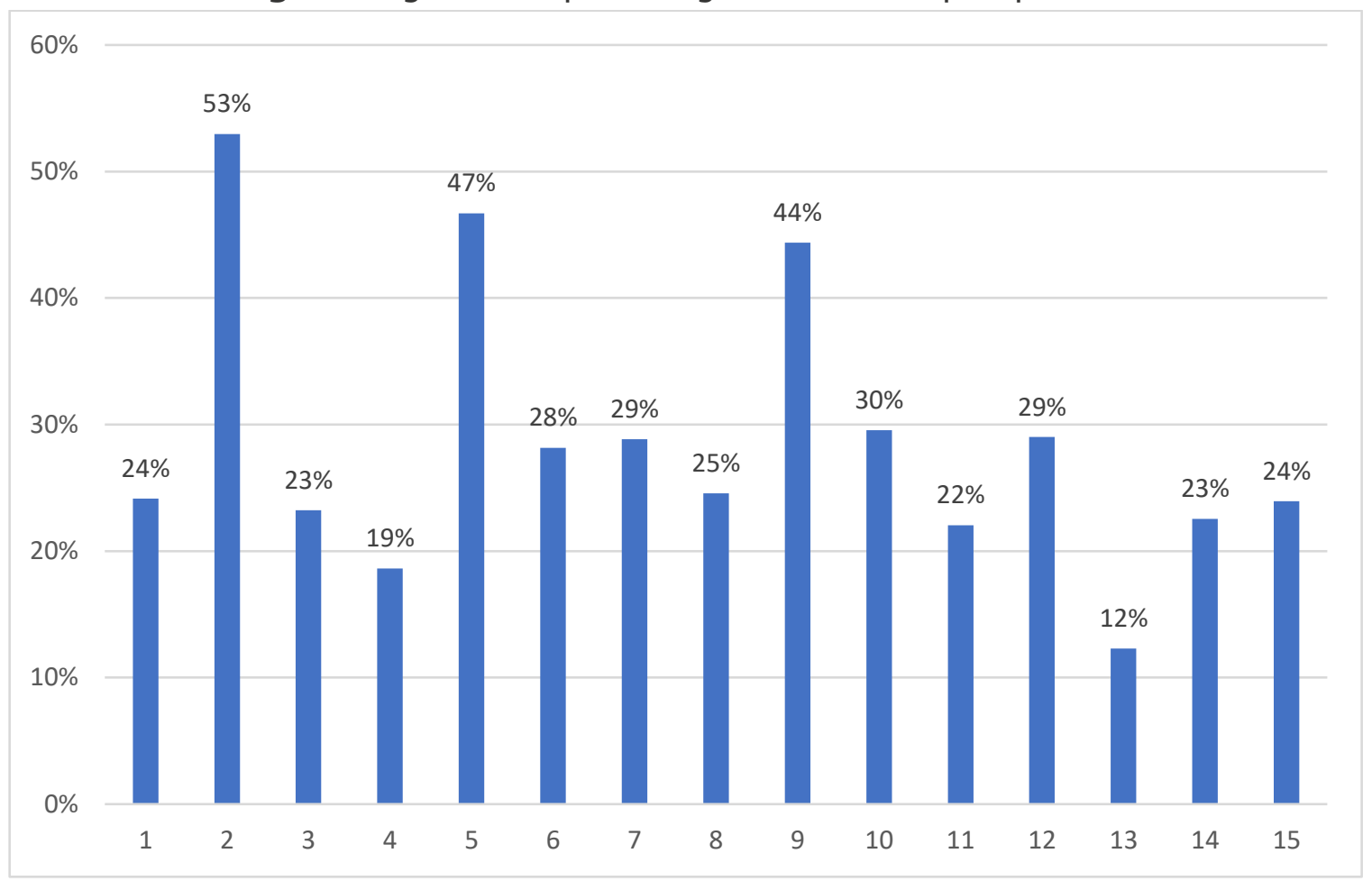

Fonte: dados da pesquisa (2017).

$\mathrm{Na}$ tabela 4 encontram-se os dados equivalentes às médias do percentual de acertos por categoria, divididas em: nove questões sobre SI e notação cientifica, três envolvendo noções básicas de cinemática e três sobre interpretação de gráficos.

Tabela 4: dados divididos por categoria do teste.

\begin{tabular}{|l|c|c|c|c|}
\hline Grupo & N & $\begin{array}{l}\text { Média do percentual } \\
\text { de acertos }\end{array}$ & $\begin{array}{l}\text { Desvio-padrão } \\
\text { da medida }\end{array}$ & $\begin{array}{l}\text { Desvio-padrão } \\
\text { da média }\end{array}$ \\
\hline Geral & 436 & 25 & 11,03 & 0,36 \\
\hline $\mathbf{1}$ a $\mathbf{9}$ & 292 & 32 & 12,27 & 0,40 \\
\hline $\mathbf{1 0}$ a $\mathbf{1 2}$ & 81 & 27 & 4,19 & 0,14 \\
\hline $\mathbf{1 3}$ a $\mathbf{1 5}$ & 59 & 20 & 6,37 & 0,21 \\
\hline
\end{tabular}

Fonte: dados da pesquisa (2017).

Em relação às questões de 1 a 9, encontramos uma média de 32\%, sendo que a que obteve maior porcentagem de acerto em todo o teste foi a questão 2 (53\%), envolvendo transformação de unidade, mas, por outro lado, a segunda em menor porcentagem de acerto foi a questão 4 (19\%), envolvendo também transformação de unidades, só que apresentando um maior grau de dificuldade. Conforme já mencionado, a questão 2 solicitava a transformação de $5.000 \mathrm{~m} \mathrm{em} \mathrm{km}$ e a questão 4, a transformação de $85,6 \mathrm{~m} / \mathrm{s}$ em km/h. A respeito das questões de 10 a 12 , envolvendo cinemática, básica obteve-se apenas um percentual de $27 \%$ de acertos, evidenciando as dificuldades dos alunos em resolver problemas simples de movimento retilíneo uniforme. Nas questões sobre interpretação de gráficos (13 a 15), o percentual foi mais baixo ainda, sendo de $20 \%$. 


\section{CONSIDERAÇÕES FINAIS}

As vivências dos autores tanto na Educação Básica quanto nos primeiros semestres de cursos de graduação de Ciências Exatas motivaram a proposição do presente trabalho, pois identifica-se que os estudantes ingressantes em cursos superiores, frequentemente, reprovam em componentes de primeiro semestre. Tal afirmação é confirmada com o levantamento realizado com alunos ingressante de 2017 do Campus Bagé da Unipampa, ao qual se constatou que menos de 16 \% dos alunos matriculados no componente curricular de Física de primeiro semestre foram aprovados (conforme dados apresentados na seção de introdução).

Dada tal situação, incialmente buscamos na literatura um teste de conhecimento para realizarmos uma avaliação sobre o desempenho de alunos do Ensino Médio da cidade de Bagé. Procuramos por testes sobre Sistema de Unidades e Cinemática, mas encontramos testes com questões com nível de conhecimento superior aos objetivos que nos propomos avaliar.

Portanto, foi elaborado, avaliado e aplicado um teste com o objetivo de identificar o desempeno dos alunos sobre questões de Sistema de Unidades, notação científica e noções básicas de Cinemática. Os resultados da aplicação mostram que o percentual de acertos foi menor que 30 \% e que apesar de se identificar uma diferença estatisticamente significativa entre os dados do primeiro e terceiro ano e entre os do primeiro e segundo ano tais diferenças não são expressivas, pois o percentual de acertos do primeiro, segundo e terceiro ano foram: $27 \%$, $29 \%$ e $30 \%$ respectivamente. Assim, os resultados encontrados evidenciam que os alunos possuem dificuldades em todos os quesitos do teste. Nas questões sobre transformação de unidades os alunos apresentaram o maior índice de acertos (53\%), mas sobre interpretação de gráficos apenas (20 \%). Esse resultado evidência o quanto os alunos apresentam dificuldades no estudo de gráficos e demonstram a necessidade de ações que visem a superação de tais dificuldades, pois segundo Araujo, Veit e Moreira (2004) propiciar condições para que os alunos aprendam a interpretá-los e utilizá-los gráficos contribui, não somente para a aprendizagem da Cinemática, mas também para a aprendizagem futura de outros conteúdos.

\section{REFERÊNCIAS}

ARAUJO, Ives S; VEIT, Eliane A; MOREIRA, Marco A. Atividades de modelagem computacional no auxílio à interpretação de gráficos da cinemática. Revista Brasileira de Ensino de Física, v. 26, n. 2, p. 179-184, 2004.

BACCON, Ana L. T; ARRUDA, Sergio M. Os saberes docentes na formação inicial do professor de física: elaborando sentidos para o estágio supervisionado. Ciência \& Educação, v. 16, n. 3, p. 507524, 2010.

BARBETA, Vagner B; ISSAO, Yamamoto. Dificuldades conceituais em física apresentadas por alunos ingressantes em um curso de engenharia. Revista Brasileira de Ensino de Física, v. 24, n. 3, p. 324-341, set., 2002.

BEICHNER, Robert. J. Testing student interpretation of kinematics graphs. American Journal of Physics, Woodbury, v. 62, n. 8, p. 750-768, Aug. 1994.

BELANÇON, Marco P. O ensino de física contextualizado ao século XXI. Revista Brasileira de Ensino de Física, v. 39, n. 4, p. 4001 (1-3), 2017. 
BULEGON, Ana M; TAROUCO, Liane M. R. Contribuições dos objetos de aprendizagem para ensejar o desenvolvimento do pensamento crítico nos estudantes nas aulas de física. Ciência \& Educação, v. 21, n. 3, p. 743-763, 2015.

COUTINHO, Renato X; FOLMER, Valderlei; PUNTEL, Robson L. Aproximando universidade e escola por meio do uso da produção acadêmica na sala de aula. Ciência \& Educação, v. 20, n. 3, p. 765783, 2014.

COSTA JUNIOR, EDIO; RODRIGUES, Erica C; SILVA, Marcus V. D; GOMES, Rita C. S; ASSÍS, Cristiano C. B. Um estudo estatístico sobre o aproveitamento em física de alunos de ensino médio e seus desempenhos em outras disciplinas. Revista Brasileira de Ensino de Física, v. 39, n. 1, p. 1401 (1-6), 2017.

COSTA, Roberta B. A; ALMEIDA, Caroline M. N; LOPES, Paulo T. C. Avaliando um ambiente virtual de aprendizagem para as aulas de ciências no nono ano a partir de percepções dos alunos. Revista Brasileira de Ensino de Ciências e Tecnologia, v. 8, n. 1, p. 184-199, jan./abr., 2015.

DIAS, Ângela M. M; NOVIKOFF, Cristina; SOUZA, Luiz E. S. Laboratórios de aprendizagem de física: resultados de uma experiência pedagógica sustentável. Física na Escola, v. 12, n. 2, p. 12-14, 2011.

GERAB, Fábio; VALÉRIO, Araceli D. A. Relação entre o desempenho em física e o desempenho em outras disciplinas da etapa inicial de um curso de engenharia. Revista Brasileira de Ensino de Física, v. 36, n. 2, p. 2401 (1-9), 2014.

GOMES, Alessandro D. T; BORGES, Antônio T; JUSTI, Rosária. Processos e conhecimento envolvidos na realização de atividades práticas: revisão da literatura e implicações para a pesquisa. Investigações em Ensino de Ciências, v. 13, n. 2, p. 187-207, 2008.

GOMES, André T; GARCIA, Isabel K. Aprendizagem significativa na EJA: uma análise da evolução conceitual a partir de uma intervenção didática com a temática energia. Investigações em Ensino de Ciências, v. 19, n. 2, p. 289-321, 2014.

LABURÚ, Carlos E; BARROS, Marcelo A. Problemas com a compreensão de estudantes em medição: razões para a formação do paradigma pontual. Investigações em Ensino de Ciências, v. 14, $n$. 2, p. 151-162, 2009.

LIMA, Kilma S; TENÓRIO, Alexandro C; BASTOS, Eloisa F. B. N. Concepções de um professor de física sobre avaliação: um estudo de caso. Ciência \& Educação, v. 16, n. 2, p. 309-322, 2010.

LUNKES, Mércio J; ROCHA FILHO, João B. A baixa procura pela licenciatura em física, com base em depoimentos de estudantes do ensino médio público do oeste catarinense. Ciência \& Educação, v. 17 , n. 1, p. 21-34, 2011.

MACHADO, Aniara R; MARQUES, Carlos A; SILVA, Rejane M. G. Sentidos e significados de problemas e a problematização em um processo de (re)planejamento coletivo de uma situação de estudo. Ciência \& Educação, v. 22, n. 1, p. 23-42, 2016.

MARTINS, Idalin; ABELHA, Marta; COSTA, Nilza; ROLDÃO, Maria C. Impacto do currículo português das ciências físicas e naturais nas práticas docentes. Ciência \& Educação, v. 17, n. 4, p. 771-788, 2011.

MACHADO, Vinicius; PINHEIRO, Nilcéia A. M. Investigando a metodologia dos problemas geradores de discussões: aplicações na disciplina de física no ensino de engenharia. Ciência \& Educação, v. 16 , n. 3, p. 525-542, 2010. 
OLIVEIRA, Ângelo M. M; MOREIRA, Marco A. Um estudo exploratório para avaliar a dificuldade de problemas em ensino de física utilizando a teoria da carga cognitiva com o auxílio de uma hipermídia. Revista Brasileira de Ensino de Ciências e Tecnologia, v. 9, n. 1, p. 26-61, jan./abr., 2016.

OLIVEIRA, Tobias E; ARAUJO, Ives S; VEIT, Eliane A. Sala de aula invertida (flipped classoom): inovando as aulas de física. Física na Escola, v. 14, n. 2, p. 4-13, 2016.

PARISOTO, Mara F; MOREIRA, Marco A; KILIAN, Alex S. Efeito da aprendizagem baseada no método de projetos e na unidade de ensino potencialmente significativa na retenção do conhecimento: uma análise quantitativa. Revista Brasileira de Ensino de Ciências e Tecnologia, v. 9, n. 2, p. 268292, mai./ago., 2016.

PROENÇA, Marcelo C; PIROLA, Nelson A. O conhecimento de polígonos e poliedros: uma análise do desempenho de alunos do ensino médio em exemplos e não exemplos. Ciência \& Educação, v. 17, n. 1, p. 199-217, 2011.

RICARDO, Elio C; FREIRE, Janaína C. A. A concepção dos alunos sobre a física no ensino médio: um estudo exploratório. Revista Brasileira de Ensino de Física, v. 29, n. 2, p. 251-266, 2007.

SANTAROSA, Maria C. P; MOREIRA, Marco A. O cálculo nas aulas de física da UFRGS: um estudo exploratório. Investigações em Ensino de Ciências, v. 16, n. 2, p. 317-351, 2011.

SANTOS, Cintia A. B; CURI, Edda. A formação dos professores que ensinam física no ensino médio. Ciência \& Educação, v. 18, n. 4, p. 837-849, 2012.

SILVEIRA, Fernando L. Determinando a significância estatística para as diferenças entre médias. Disponível em: https://www.if.ufrgs.br/ lang/Textos/Comparacoes em media.pdf. Acesso em: 16 set. 2019.

SILVEIRA, F. L.; MOREIRA, M. A.; AXT, R. Validação de um teste para verificar se o aluno possui concepções científicas sobre corrente elétrica em circuitos simples. Ciência e Cultura, São Paulo, v. 41, n. 11, p. 1129-1133, nov. 1989. 


\section{Apêndice A}

\section{IMPORTANTE: NÃO FAÇA MARCAS NAS FOLHAS DE QUESTÕES RESPONDA APENAS NA FOLHA DE RESPOSTAS}

Teste sobre conhecimentos básicos de Física e transformação de unidades.

Este teste é constituído por 15 questões de múltipla escolha com quatro alternativas cada uma. Dentre as alternativas, escolha apenas uma, a que melhor responde à questão, assinalando-a na grade em anexo.

Importante: os gráficos presentes neste teste foram construídos de acordo com um sistema cartesiano ortogonal de coordenadas, conforme a figura abaixo.

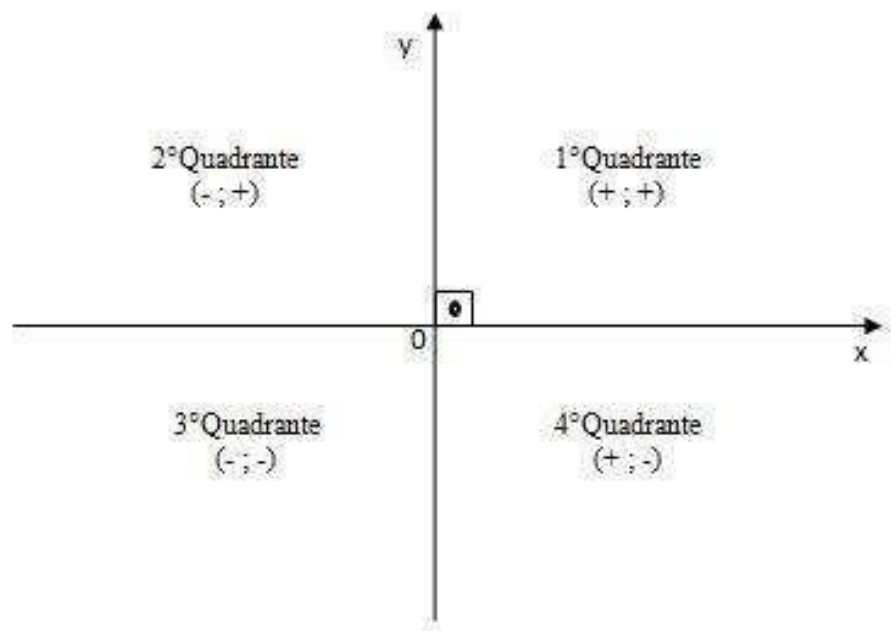

1) Transforme $5 \mathrm{~g}$ em kg:
a) $5,0 \times 10^{-2} \mathrm{~kg}$.
b) $5,0 \times 10^{-3} \mathrm{~kg}$.
c) $5,0 \times 10^{-5} \mathrm{~kg}$.
d) $5,0 \times 10^{2} \mathrm{~kg}$.

2) Transforme $5.000 \mathrm{~m}$ em km:
a) 5,0 km.
b) $5,0 \times 10^{-3} \mathrm{~km}$.
c) $5,0 \times 10^{-2} \mathrm{~km}$.
d) $5,0 \times 10^{1} \mathrm{~km}$.

3) Transforme 2 km/h em m/s:
a) $5,60 \times 10^{1} \mathrm{~m} / \mathrm{s}$.
b) $5,60 \times 10^{-1} \mathrm{~m} / \mathrm{s}$.
c) $7,20 \times 10^{2} \mathrm{~m} / \mathrm{s}$.
d) $7,20 \times 10^{3} \mathrm{~m} / \mathrm{s}$.

4) Transforme $85,6 \mathrm{~m} / \mathrm{s}$ em km/h:
a) $31,0 \times 10^{2} \mathrm{~km} / \mathrm{h}$. b) $24,0 \times 10^{0} \mathrm{~km} / \mathrm{h}$.
c) $21,0 \times 10^{-1} \mathrm{~km} / \mathrm{h}$.
d) $31,0 \times 10^{1} \mathrm{~km} / \mathrm{h}$.

Assinale a alternativa que representa os símbolos das unidades de tempo, massa e força respectivamente:

a) J, kg e s.

b) $\quad \mathrm{kg}$, s e m. 
c) $\mathrm{J}, \mathrm{m} / \mathrm{s}$ e $\mathrm{N}$.

d) $\quad \mathrm{s}, \mathrm{kg}$ e N.

As questões 6), 7) e 8) devem ser respondidas com base no cubo da figura 1. As respostas devem estar em notação científica.

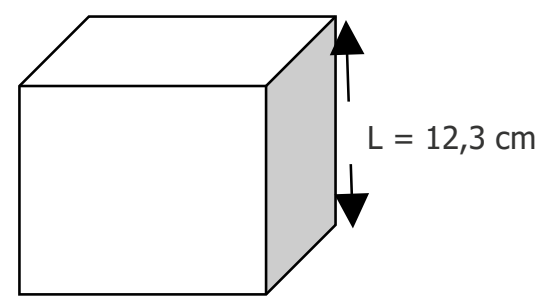

Figura 1

6) O lado do quadrado em quilômetros, metros e milímetros vale respectivamente:

a) $1,23 \times 10^{-2} \mathrm{~km}, 1,23 \times 10^{2} \mathrm{~m}$ e $1,23 \times 10^{-2} \mathrm{~mm}$.

b) $\quad 1,23 \times 10^{-4} \mathrm{~km}, 1,23 \times 10^{-1} \mathrm{~m}$ e $1,23 \times 10^{2} \mathrm{~mm}$.

c) $\quad 1,23 \times 10^{-4} \mathrm{~km}, 1,23 \times 10^{1} \mathrm{~m} \mathrm{e} 1,23 \times 10^{1} \mathrm{~mm}$.

d) $1,23 \times 10^{-3} \mathrm{~km}, 1,23 \times 10^{-1} \mathrm{~m} \mathrm{e} 1,23 \times 10^{2} \mathrm{~mm}$.

7) O volume do cubo em metros cúbicos é?
a) $\quad 1,86 \times 10^{-4} \mathrm{~m}^{3}$.
b) $1,86 \times 10^{-3} \mathrm{~m}^{3}$.
c) $\quad 1,86 \times 10^{-5} \mathrm{~m}^{3}$.
d) $\quad 1,86 \times 10^{2} \mathrm{~m}^{3}$.

8) O volume em quilômetros cúbicos é?
a) $\quad 1,86 \times 10^{-14} \mathrm{~km}^{3}$.
b) $\quad 1,86 \times 10^{12} \mathrm{~km}^{3}$.
c) $\quad 1,86 \times 10^{-12} \mathrm{~km}^{3}$.
d) $1,86 \times 10^{11} \mathrm{~km}^{3}$. 
9) João vai de sua casa até a escola de ônibus, o trajeto é de 1 h20 para chegar. Quanto tempo João leva em segundos para chegar ao seu destino.
a) $28.800 \mathrm{~s}$.
b) $3.820 \mathrm{~s}$.
c) $4.800 \mathrm{~s}$.
d) $3.600 \mathrm{~s}$.

10) Considerando que a equação horária de um movimento retilíneo é $x(t)=60+20 t$ é correto afirmar que:
a) No instante $6 \mathrm{~s}$ a posição do objeto vale $60 \mathrm{~m}$.
b) $\mathrm{O}$ valor numérico de 60 tem unidade de comprimento e o de 20 unidade de velocidade.
c) O valor numérico de 60 representa a velocidade do objeto e o valor numérico de 20 representa a aceleração do objeto.
d) No instante $t=3 \mathrm{~s}$ a posição do objeto é $100 \mathrm{~m}$.

11) Na tabela abaixo são mostradas as velocidades de um carro em um desafio de arrancadas, nos 30s iniciais da corrida:

\begin{tabular}{|l|l|}
\hline$t(s)$ & $v(\mathrm{~m} / \mathrm{s})$ \\
\hline 0 & 0 \\
\hline 10 & 20 \\
\hline 20 & 40 \\
\hline 30 & 60 \\
\hline
\end{tabular}

Em relação à tabela é incorreto afirmar que:
a) Ao longo dos 30s, a aceleração permanece constante.
b) Ao longo dos 30s, a velocidade aumentou $2 \mathrm{~m} / \mathrm{s}$ a cada segundo.
c) O deslocamento do carro no instante de 10 a 20s é igual ao deslocamento ocorrido entre os instantes de 20 a 30 s.

d) O deslocamento do carro no instante de 10 a 20s é menor que o deslocamento ocorrido entre os instantes de 20 a 30 s. 
12) Um bloco é deslocado com uma velocidade constante de $5 \mathrm{~m} / \mathrm{s}$. Qual é a distância percorrida pelo bloco após 20 segundos, 30 minutos e quando completar uma hora de movimento?

a) $100 \mathrm{~m}, 10 \mathrm{~km}, 20 \mathrm{~km}$.

b) $100 \mathrm{~m}, 9 \mathrm{~km}, 18 \mathrm{~km}$.

c) $\quad 50 \mathrm{~m}, 9 \mathrm{~km}, 18 \mathrm{~km}$.

d) $50 \mathrm{~m}, 10 \mathrm{~km}, 20 \mathrm{~km}$.

13) (BERNAL e YAMAMOTO, 2002). No gráfico seguinte é mostrada a velocidade de um objeto em função do tempo.

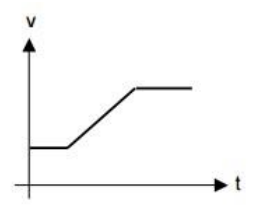

Qual dos gráficos abaixo mostra a relação entre a força resultante e o tempo?

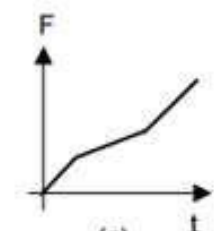

(a)

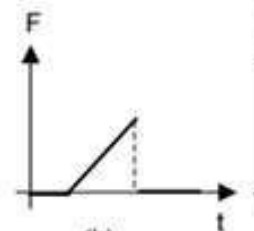

(b)

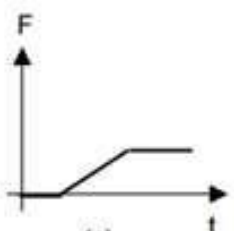

(c)

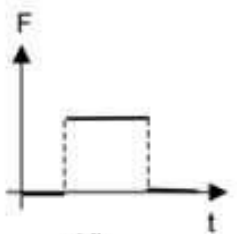

(d)

14) (JÚNIOR, 2017). O gráfico seguinte representa o movimento de um objeto. Qual das sentenças é a melhor interpretação desse movimento?

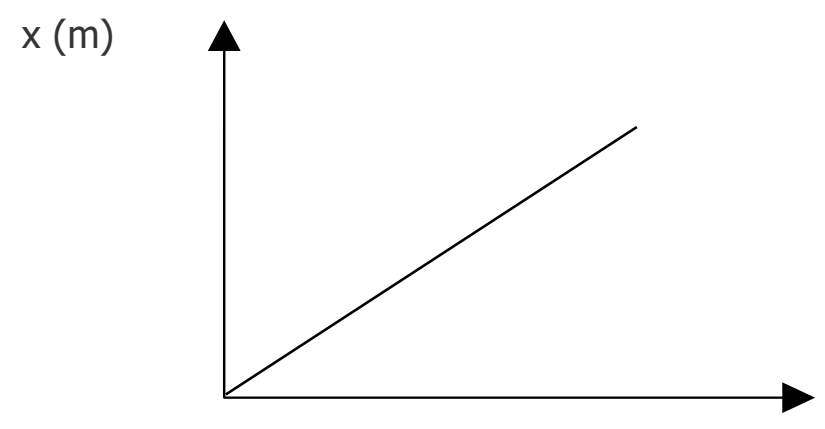

$t(s)$

a) O objeto está se movendo com uma aceleração constante e diferente de zero.

b) O objeto não se move.

c) O objeto está se movendo com uma velocidade que aumenta uniformemente.

d) O objeto está se movendo com uma velocidade constante. 
15) Uma bola é abandonada da cobertura de um prédio de $40 \mathrm{~m}$ de altura. Qual gráfico pode representar qualitativamente o comportamento da velocidade em função do tempo. Considere que a bola se desloca somente na direção vertical e se desprezam as forças resistivas (ação somente na força gravitacional).

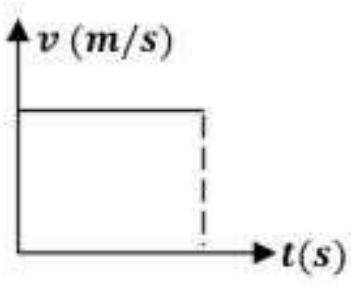

a

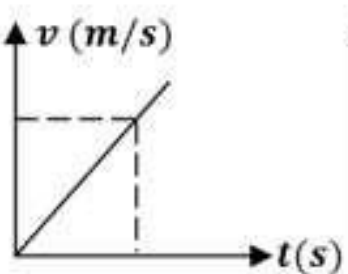

b
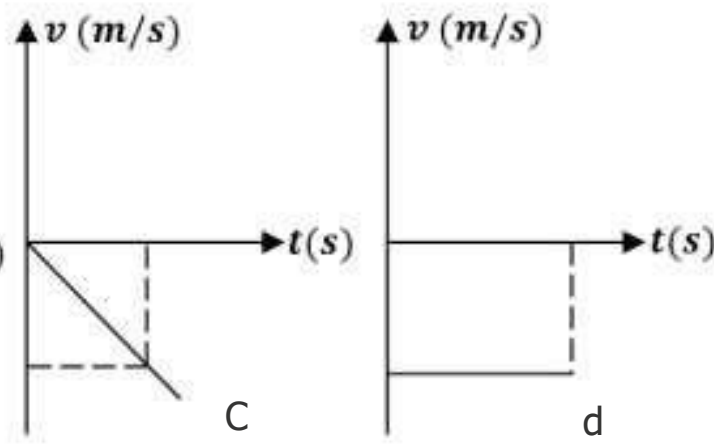

\section{Referências}

Araujo, I. S.; Veit, E. A. \& Moreira, M. A. (2002) - Adaptado de Beichner, R. J. Am. J. Phys. 62, 750 (1994). Interpretação de gráficos da Cinemática. Disponível em:

http://www.if.ufrgs.br/cref/ntef/La_Paz/La_Paz2/Teste_inicial.pdf. Acesso em: 30 maio. 2017.

BERNAL, Vagner Barbeta.; YAMAMOTO, Issao. Dificuldades Conceituais em Física Apresentadas por Alunos Ingressantes em um Curso de Engenharia. Disponível em: http://www.scielo.br/pdf/rbef/v24n3/a11v24n3.pdf. Acesso em: 30 maio. 2017.

HALLIDAY D.; RESNICK R.; WALKER J. Fundamentos de Física: Mecânica, v. 1. $8^{a}$ edição. Editora LTC, 2009.

XAVIER, Claudio; BENIGNO, Barreto. Física aula por aula: Mecânica. v. 1. 1ª edição. Editora FTD, 2010.

JÚNIOR, Joab Silas da Silva. Exercícios Sobre Gráfico Do Movimento Uniforme. Disponível em: http://exercicios.mundoeducacao.bol.uol.com.br/exercicios-fisica/exercicios-sobre-graficomovimentouniforme.htm\#resposta-3990. Acesso em: 30 maio. 2017.

MESQUITA, Rosa Maria. Teste entre relação de força e movimento. Disponível em: http://lief.if.ufrgs.br/pub/cref/n29_Rosa/arquivos/teste_inicial_final.pdf. Acesso em: 30 maio. 2017. 


\section{Grade de Respostas}

Nome:

Turma:

\begin{tabular}{|c|c|c|c|c|}
\hline & $\mathbf{A}$ & B & C & D \\
\hline 1 & & & & \\
\hline 2 & & & & \\
\hline 3 & & & & \\
\hline 4 & & & & \\
\hline 5 & & & & \\
\hline 6 & & & & \\
\hline 7 & & & & \\
\hline 8 & & & & \\
\hline 9 & & & & \\
\hline 10 & & & & \\
\hline 11 & & & & \\
\hline 12 & & & & \\
\hline 13 & & & & \\
\hline 14 & & & & \\
\hline 15 & & & & \\
\hline
\end{tabular}

